\title{
SSSPM J1444-2019: An extremely high proper motion, ultracool subdwarf ${ }^{\star}$
}

\author{
R.-D. Scholz ${ }^{1}$, N. Lodieu ${ }^{1,2}$, and M. J. McCaughrean ${ }^{1,3}$ \\ 1 Astrophysikalisches Institut Potsdam, An der Sternwarte 16, 14482 Potsdam, Germany \\ e-mail: rdscholz@aip.de \\ 2 University of Leicester, Department of Physics \& Astronomy, University Road, Leicester LE1 7RH, UK \\ e-mail: nl41@star.le.ac.uk \\ ${ }^{3}$ University of Exeter, School of Physics, Stocker Road, Exeter EX4 4QL, UK \\ e-mail:mjm@aip.de, mjm@astro.ex.ac.uk
}

Received 19 October 2004 / Accepted 30 October 2004

\begin{abstract}
We present the discovery of a new extreme high proper motion object ( $\sim 3.5$ arcsec/year) which we classify as an ultracool subdwarf with $[\mathrm{M} / \mathrm{H}] \sim-0.5$. It has a formal spectral type of sdM9 but also shows L-type features: while the VO bands are completely absent, it exhibits extremely strong TiO absorption in its optical spectrum. With a radial velocity of about $-160 \mathrm{~km} \mathrm{~s}^{-1}$ and a rough distance estimate of $16-24 \mathrm{pc}$, it is likely one of the nearest halo members crossing the Solar neighbourhood with a heliocentric space velocity of $(U, V, W)=(-244,-256,-100) \pm(32,77,6) \mathrm{km} \mathrm{s}^{-1}$.
\end{abstract}

Key words. surveys - astrometry - stars: kinematics - stars: low-mass, brown dwarfs - subdwarfs - solar neighbourhood

\section{Introduction}

It is well understood that the observed magnitudes of stars cannot be taken as direct indicator of their true luminosities without accounting for their distances. Similarly, the distance must be known before their measured proper motions on the celestial sphere can be converted into their true velocities. Thus, high proper motion (hereafter HPM) objects represent a mixture of very nearby stars, neighbours of the Sun in the local Galactic spiral arm, and very fast representatives of the Galactic thick disk and halo, just passing through the neighbourhood. As a consequence, even though thick disk and halo stars are relatively rare when compared to the number density of stars in the Solar neighbourhood, they are over-represented in HPM samples (Digby et al. 2003).

In HPM works such as the Luyten Half Second (LHS) catalogue (Luyten 1979), the stellar proper motions are listed in units of arcsec/yr, even though the majority of stars in those catalogues have proper motions below $1 \mathrm{arcsec} / \mathrm{yr}$. In the LHS, only about 530 sources exceed that limit, although an additional 36 have been discovered during the last 15 years, suggesting that the sample is by no means yet complete (cf. Table 1 of Subasavage et al. 2004). At the more extreme end, there are just 25 LHS stars with proper motions between 3.5 and $10.3 \mathrm{arcsec} / \mathrm{yr}$, but there again the sample is incomplete: after decades of stagnation, three new members have

^ Based on archival data from the SuperCOSMOS Sky Surveys, 2MASS, and DENIS, and observations with the ESO 3.6-m telescope (ESO 072.C-0630) and VLT (ESO 072.C-0725). recently been added to this elite club, namely the red dwarf star SO $025300.5+165258$ with $\mu \sim 5.1$ arcsec/yr (Teegarden et al. 2003) and the brown dwarf binary $\varepsilon$ Indi Ba,Bb with $\mu \sim 4.7 \mathrm{arcsec} / \mathrm{yr}$ (Scholz et al. 2003; McCaughrean et al. 2004). These latter two objects are also the first brown dwarfs known in the immediate Solar neighbourhood, i.e. within a horizon of about 4 pc.

In this letter, we present the discovery of a new object with $\sim 3.5 \mathrm{arcsec} / \mathrm{yr}$ proper motion, fainter and redder than all other known extreme HPM objects apart from the brown dwarfs $\varepsilon$ Indi Ba,Bb (Sect. 2). We describe low- and high-resolution spectroscopic follow-up observations which have allowed us to classify the object and to measure its radial velocity (Sect. 3). Based on all the available data, we discuss the likely status of the object as one of the nearest cool halo subdwarfs (Sect. 4).

\section{Proper motion measurement and photometry}

SSSPM J1444-2019 was discovered in the course of our ongoing HPM survey of the southern sky using multi-epoch optical data from the SuperCOSMOS Sky Surveys (SSS; Hambly et al. 2001a,b,c) and near-infrared data from the Two-Micron All Sky Survey (2MASS; Cutri et al. 2003). After the $\varepsilon$ Indi Ba,Bb system it has the second largest proper motion among the objects we have found to date. The search strategy which led to the discovery of SSSPM J1444-2019 is briefly described here.

First, we selected bright $(J<14)$ objects in the 2MASS database without an optical counterpart, working on the basis that an epoch difference of $\sim 15$ years between the 2 MASS 

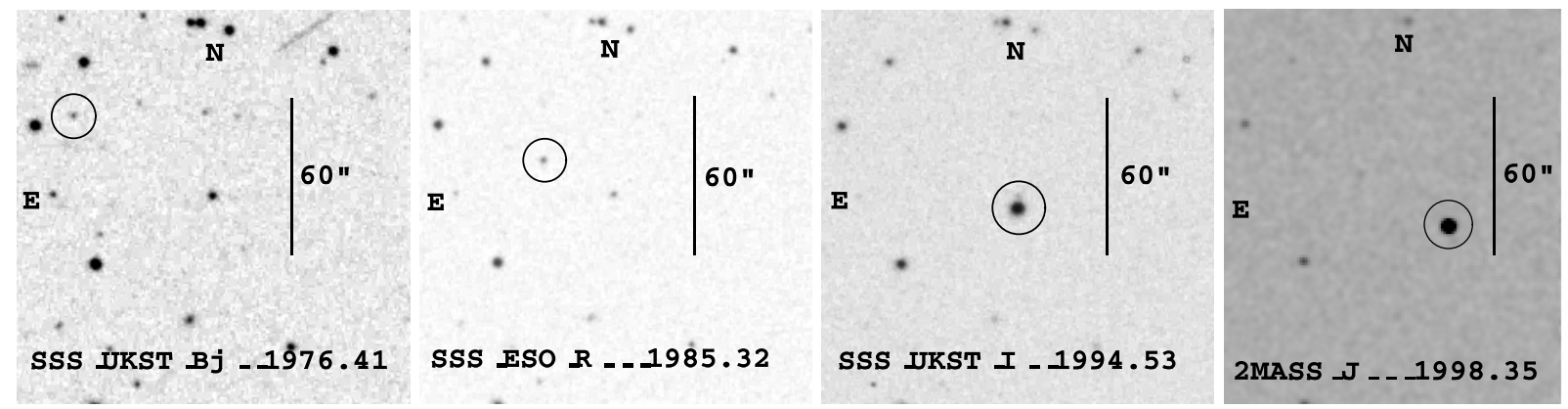

Fig. 1. Finding charts of SSSPM J1444-2019 (circled) at different epochs clearly illustrating its extremely large proper motion. The different passband data are sorted according to the epochs at which they were taken, from blue and red SSS plates to the most recent epoch 2MASS $J$ band image, and also demonstrate the very red optical and optical-to-infrared colours of the object (see also Table 1). North is up and East left.

observations and the optical A2.0 catalogue (Monet et al. 1998) in the southern sky might mean the lack of cross-identification is caused by a large proper motion of the object. In order to exclude crowded fields and the Galactic plane, we restricted our search to higher Galactic latitudes $\left(|b|>20^{\circ}\right)$ and selected only isolated 2MASS sources with no neighbours inside a 30 arcsec radius. About $\sim 11000$ candidates were extracted in this manner and this sample was then checked for true HPM objects using SSS finding charts in three passbands at four epochs. The success rate was $\sim 3 \%$, with most of the confirmed objects turning out to be previously known HPM stars.

For each new HPM object, the accuracy of the proper motion determination was improved by using all available SSS positions (including those from overlapping plates), the 2MASS position, and additional epoch data from the DEep Near-Infrared Survey (DENIS; Epchtein et al. 1997) if available. The huge proper motion of SSSPM J1444-2019 was reason alone for follow-up observations, but in addition, its very red optical and optical-to-infrared colours but rather blue $J-K_{\mathrm{s}}$ colour (Table 1) triggered special attention and led us to speculate that it was a potential subdwarf.

\section{Spectroscopy}

Low-resolution optical spectroscopy was obtained on 16 March 2004 for SSSPM J1444-2019 and two comparison objects (spectral types M9.5 and L2) with EFOSC2 mounted on the ESO 3.6-m telescope in service mode (ESO programme 072.C-0630). Using grism\#5, the wavelength coverage was 5200-9350 ̊ with a $F W H M$ resolution of $\sim 12.8 \AA$. The exposure times were 900s, 480s, and 600s for SSSPM J1444-2019, LP 944-20, and SSSPM J0829-1309, respectively. The spectral extraction, wavelength, and flux calibration were made using standard MIDAS routines. These spectra are shown in Fig. 2 along with an additional comparison spectrum (spectral type sdM9.5) obtained by Scholz et al. (2004) observed with EMMI on the ESO NTT in May 2003. The resolution of the latter spectrum was $\sim 5 \AA$ over the wavelength range 3850-9100 $\AA$.

High-resolution $(R \sim 16000$ at $6000 \AA)$ optical (5850$7400 \AA$ ) spectroscopy of SSSPM J1444-2019 was obtained using FORS2 on the ESO VLT, along with a corresponding spectrum of LP 944-20 (Tinney 1998) to serve as a comparison object (Fig. 3). The observations were carried out in service

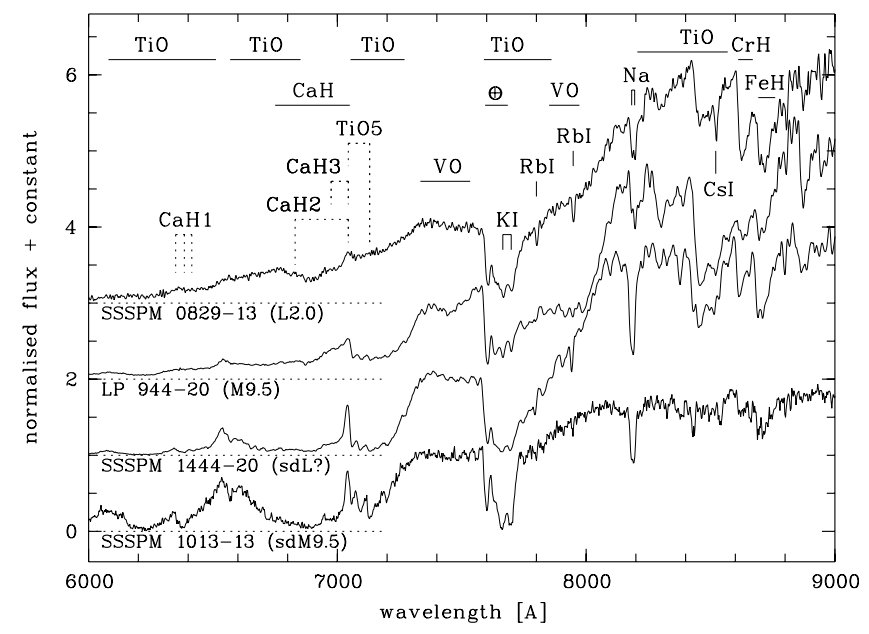

Fig. 2. Optical spectra taken with EFOSC2 on the ESO 3.6-m telescope for SSSPM J1444-2019, LP 944-20, and SSSPM J0829-1309, along with an NTT/EMMI spectrum of the latest known M-type subdwarf (sdM9.5) SSSPM J1013-1356 (Scholz et al. 2004). The spectra are normalised at $7500 \AA$. Key spectral features of $\mathrm{M}$ and $\mathrm{L}$ (sub)dwarfs are labeled. Also shown are the spectral regions defining the spectral indices $\mathrm{TiO} 5, \mathrm{CaH} 1, \mathrm{CaH} 2$, and $\mathrm{CaH} 3$ used in the Gizis (1997) subdwarf classification scheme. Uncorrected telluric absorption is indicated by the circled plus sign.

mode on two different nights (29 August 2003 and 17 December 2003) using grism GRIS_1200R+93 with a slit width of 1 arcsec: the seeing was better than 0.8 arcsec during both observations. The total integration times were $1800 \mathrm{~s}$ and 840 s for SSSPM J1444-2019 and LP 944-20, respectively. Data reduction was performed using standard IRAF tools and arc lamp wavelength calibration yielded an accuracy better than $0.2 \AA$.

The FORS2 service observing scheme did not include the measurement of radial velocity $(R V)$ standards during the same nights as our observations, but we have been able to use the comparison spectrum of LP 944-20, which has a known $R V$ of $+7.4 \pm 1.3 \mathrm{~km} \mathrm{~s}^{-1}$ (Tinney \& Reid 1998). By cross-correlating the two spectra shown in Fig. 3, we were able to measure a relative $R V$ for SSSPM J1444-2019 of $-149.2 \pm 3.8 \mathrm{~km} \mathrm{~s}^{-1}$ with respect to LP 944-20. After correcting for the Earth's orbital motion during the different observing nights, a heliocentric $R V$ estimate for SSSPM J1444-2019 of $-156.3 \pm 8.8 \mathrm{~km} \mathrm{~s}^{-1}$ was 
Table 1. Astrometry and photometry from SSS, 2MASS, and DENIS.

\begin{tabular}{|c|c|c|c|c|c|c|c|c|c|c|}
\hline Name & $\alpha, \delta$ & Epoch & $\mu_{\alpha} \cos \delta$ & $\overline{\mu_{\delta}}$ & $B_{J}$ & $R$ & $I$ & $\bar{J}$ & $H$ & $K_{\mathrm{s}}$ \\
\hline SSSPM J... & $(\mathrm{J} 2000)$ & & \multicolumn{2}{|c|}{$\operatorname{mas} / \mathrm{yr}$} & & (SSS) & & & (2MASS) & \\
\hline $1444-2019$ & $144420.67-201922.2$ & 1998.351 & $-2919 \pm 9$ & $-1950 \pm 5$ & 21.159 & 18.569 & 14.954 & 12.546 & 12.142 & 11.933 \\
\hline
\end{tabular}

Notes: Coordinates are from 2MASS, which provides accurate positions at the most recent epoch. SSS magnitudes are mean values. The proper motion was determined from one 2MASS and six SSS positions. The addition of two DENIS measurements (mean $I, J, K=14.945,12.485$, 11.891; epoch 2000.397) to the proper motion fit led to a less accurate solution: $(-2954,-1977) \pm(24,16) \mathrm{mas} / \mathrm{yr}$.

obtained, with the final error dominated by the $0.2 \AA$ accuracy of our wavelength calibration.

The spectrum of SSSPM J1444-2019 is remarkable in several aspects. Overall, it is much redder than that of the latest known M-type subdwarf SSSPM 1013-1356 (sdM9.5; bottom of Fig. 2) and comparable to that of the field brown dwarf LP 944-20 (Tinney 1998), for which we adopted the spectral type M9.5 determined by Reid et al. (2002). The TiO absorption bands are extremely strong, as are the $\mathrm{CaH}$ bands, indicating a cool subdwarf nature. The complete absence of the VO bands, strong $\mathrm{CrH}$ and $\mathrm{FeH}$, and in particular the presence of the atomic Rb I lines, are typical of L dwarfs, as can be seen in the spectrum of the L2 dwarf SSSPM J0829-1309 shown in Fig. 2 (Scholz \& Meusinger 2002).

The higher-resolution spectrum of SSSPM J1444-2019 shows no $\mathrm{Li}$ absorption at $6708 \AA$, while it is clearly visible in the known brown dwarf LP 944-20 when observed with the same instrument (Fig. 3). There is apparent $\mathrm{H} \alpha$ line emission in SSSPM J1444-2019, despite the fact that it is not usually observed in cool subdwarfs. However, it is not quite at the right wavelength, being shifted by about $-2 \AA$ with respect to the expected location after taking into account the $R V$, and this line may in fact be a blend of other, unrelated spectral features.

We have applied the Gizis (1997) classification scheme for $\mathrm{K}$ and $\mathrm{M}$ subdwarfs to the SSSPM J1444-2019 spectra. The spectral indices $\mathrm{TiO} 5, \mathrm{CaH} 1, \mathrm{CaH} 2$, and $\mathrm{CaH} 3$ were measured to be $0.08,0.42,0.11$, and 0.23 , respectively from the lowresolution spectrum, and $0.079,0.473,0.120$, and 0.232 , respectively, from the high-resolution spectrum. The TiO5 and $\mathrm{CaH} 2$ indices are so small that they fall outside the plotted range in Fig. 1 of Gizis (1997), while the CaH1 and CaH3 values are very close to the limits. The $\mathrm{CaH} 2$ and $\mathrm{CaH} 3$ indices for SSSPM J1444-2019 are very similar to those measured for the sdM9.5 object SSSPM 1013-1356 (Scholz et al. 2004), while the TiO5 index has the smallest ever measured value. Conversely, the SSSPM J1444-2019 CaH1 index is larger than that of SSSPM 1013-1356, but this index is not very useful for the latest-type objects according to Gizis (1997).

Applying the classification scheme of Gizis (1997), which is formally valid only to $\mathrm{sdM} 7$, to the extreme spectral indices of SSSPM J1444-2019, we compute a spectral type of sdM9. On the other hand, the L-type features and the very red continuum mentioned above would also allow us to classify this object as an early-L subdwarf, similar to LSR 1610-0040 (Lépine et al. 2003). However, the Gizis (1997) scheme yielded only sdM6.0 for this latter source, completely inconsistent with its much later spectral energy distribution.



Fig. 3. FORS2 spectra of SSSPM J1444-2019 and the brown dwarf LP 944-20. The spectral region employed in the Gizis (1997) subdwarf classification scheme is shown. The Li absorption line at $6708 \AA$ in LP 944-20 and some of the sharp spectral features (mainly TiO) used in the radial velocity determinations are marked. Telluric absorption is indicated by a circled plus sign.

\section{Discussion}

Most of the known extreme HPM ( $>3.5$ arcsec/yr) objects are very nearby $(d<10 \mathrm{pc})$, the only exceptions being the DA7 white dwarf LHS 56 at $\sim 16 \mathrm{pc}$ and the K-type halo subdwarfs LHS 52/53, a wide binary ( $~ 8700 \mathrm{AU}$ separation) at $\sim 29 \mathrm{pc}$. How far away is the newly discovered HPM ultracool subdwarf SSSPM J1444-2019?

With the discovery of an increasing number of ultracool subdwarfs, mostly via HPM surveys, it has become possible to explore an extension of the subdwarf classification scheme into the late-M, L, and T dwarf regimes (see, e.g., Burgasser et al. 2004), but as yet, there is no formal scheme which covers SSSPM J1444-2019. Thus it is hard to predict its intrinsic luminosity and hence distance; in addition, there are no trigonometric parallaxes available for similar objects, making direct bootstrapping difficult.

For a first guess, we can compare the $I-J$ and $J-$ $K_{\text {s }}$ colours of SSSPM J1444-2019 with those predicted by models of $8-10 \mathrm{Gyr}$ old, low-metallicity objects (Baraffe et al. 1997, 1998). In Fig. 4, we see that the colours of SSSPM J1444-2019, the early-L subdwarf LSR 1610-0040, and the late-L subdwarf 2MASS J0532+8246 (Burgasser et al. 2003) are all rather consistent with moderately low metallicity models $([\mathrm{M} / \mathrm{H}]=-0.5)$. Concentrating on just 


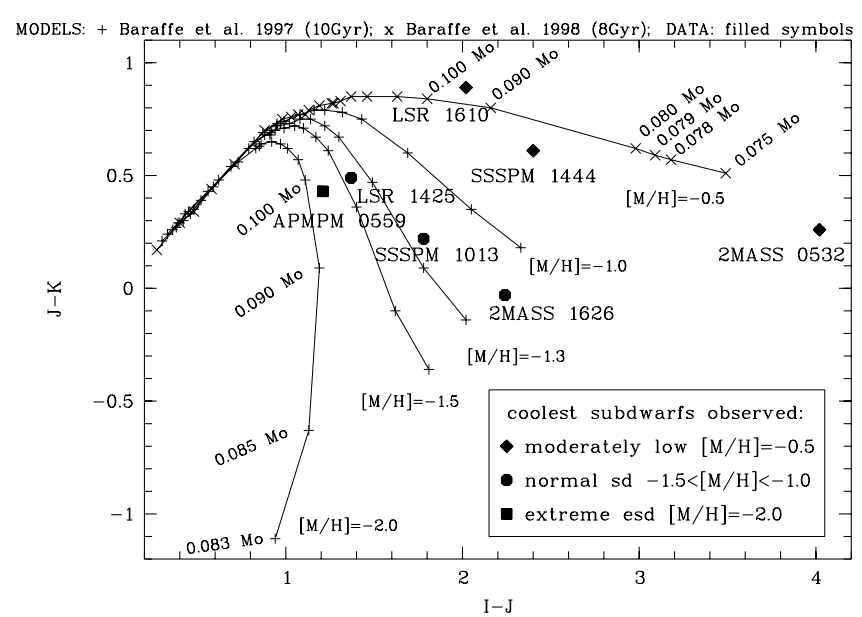

Fig. 4. Colour-colour diagram for the coolest known subdwarfs compared with evolutionary models (Baraffe et al. 1997, 1998). SSSPM J1444-2019, LSR 1610-0040, and 2MASS J0532+8246 all appear more consistent with moderately low-metallicity models than with their normal or extreme subdwarf counterparts.

SSSPM J1444-2019 and LSR 1610-0040, Fig. 4 also shows that the models predict masses of $\sim 0.085$ and $0.095 M_{\odot}$, respectively. Then by comparing the model predictions for the absolute magnitudes for sources with these masses with the measured apparent magnitudes for SSSPM J1444-2019 and LSR 1610-0040, we can calculate nominal distances of $20 \pm 4 \mathrm{pc}$ and $30 \pm 5 \mathrm{pc}$, respectively, conservatively including 0.4 mag uncertainties. Finally, we can then compute heliocentric space velocities following Johnson \& Soderblom (1987), yielding $(U, V, W)=(-244,-256,-100) \pm(32,77,6) \mathrm{km} \mathrm{s}^{-1}$ for SSSPM J1444-2019 and $(-60,-224,-79) \pm(15,41,9) \mathrm{km} \mathrm{s}^{-1}$ for LSR 1610-0040. The space velocity of the former is clearly consistent with membership in the Galactic halo population, while that of the latter is typical of thick disk objects (Chiba \& Beers 2000).

As discussed above, the formal classification of SSSPM J1444-2019 is sdM9, although it appears likely that it is, in fact, an L-type subdwarf. The most convincing halo L subdwarf to date, 2MASS J0532+8246, has a characteristic late L-type optical spectrum, but with unusually strong $\mathrm{TiO}$ bands and a blue near-infrared colour, remarkably similar in sum to SSSPM J1444-2019. Burgasser (2004) has also reported the discovery of another L subdwarf, 2MASSJ1626+3925, which seems to extend the normal subdwarf sequence (with metallicities $-1.5<[\mathrm{M} / \mathrm{H}]<-1.0$ ) beyond SSSPM 1013-1356 (sdM9.5) into the L regime (Fig. 4).

As one of the highest proper motion sources discovered in recent years and probably one of the nearest ultracool subdwarfs, SSSPM J1444-2019 should be the subject of detailed follow-up observations and is a promising target for trigonometric parallax programmes. Further investigations of this kind are planned, including a comparison of our higher-resolution spectrum with model spectra in order to further quantify the metallicity and chemical composition of SSSPM J1444-2019.
Acknowledgements. This research is based on data from the SuperCOSMOS Sky Surveys (SSS) at the Wide-Field Astronomy Unit of the Institute for Astronomy, University of Edinburgh. We have also used data products from the 2MASS, a joint project of the University of Massachusetts and the Infrared Processing and Analysis Center/California Institute of Technology, funded by the NASA and NSF, and from the DENIS. We would like to thank Linda Schmidtobreick and Ivo Saviane, the observers who took our ESO 3.6-m low-resolution spectroscopic observations in service mode, and the EFOSC2 support astronomer, Gaspare Lo Curto, for their efforts. We would also like to thank Mario van den Ancker for his advice during Phase II preparations for our higher-resolution VLT FORS2 service observations and for his support in their scheduling. Finally, we thank Isabelle Baraffe and Gilles Chabrier for very helpful discussions.

\section{References}

Baraffe, I., Chabrier, G., Allard, F., \& Hauschildt, P. H. 1997, A\&A, 327,1054

Baraffe, I., Chabrier, G., Allard, F., \& Hauschildt, P. H. 1998, A\&A, 337, 403

Burgasser, A. J. 2004, ApJ, 614, L73

Burgasser, A. J., Kirkpatrick, J. D., Burrows, A., et al. 2003, ApJ, 592, 1186

Burgasser, A. J., Kirkpatrick, J. D., \& Lépine, S. 2004, in Proc. 13th Cool Stars Workshop, ed. F. Favata, in press [arXiv:astro-ph/0409178]

Chiba, M., \& Beers, T. 2000, AJ, 119, 2843

Cutri, R. M., Skrutskie, M. F., Van Dyk, S., et al. 2003, The 2MASS All-Sky Catalog of Point Sources, University of Massachusetts and IPAC/California Institute of Technology

Digby, A. P., Hambly, N. C., Cooke, J. A., et al. 2003, MNRAS, 344, 583

Epchtein, N., de Batz, B., Capoani, L., et al. 1997, Messenger, 87, 27

Gizis, J. E. 1997, AJ, 113, 806

Hambly, N. C., MacGillivray, H. T., Read M. A., et al. 2001a, MNRAS, 326, 1279

Hambly, N. C., Irwin, M. J., \& MacGillivray, H. T. 2001b, MNRAS, 326,1295

Hambly, N. C., Davenhall, A. C., Irwin, M. J., \& MacGillivray, H. T. 2001c, MNRAS, 326, 1315

Johnson, D. R. H., \& Soderblom, D. R. 1987, AJ, 93, 864

Lépine, S., Rich, R. M., \& Shara, M. M. 2003, ApJ, 591, L49

Luyten, W. J. 1979, LHS Catalogue, Second Edition (Minneapolis: University of Minnesota Press)

McCaughrean, M. J., Close, L. M., Scholz, R.-D., et al. 2004, A\&A, 413, 1029

Monet, D., Bird, A., Canzian, B., et al. 1998, The USNO-A2.0 Catalogue, US Naval Observatory Flagstaff Station (USNOFS) and Universities Space Research Association (USRA) stationed at USNOFS

Reid, I. N., Kirkpatrick, J. D., Liebert, J., et al. 2002, AJ, 124, 519

Scholz, R.-D., Meusinger, H. 2002, MNRAS, 336, L49

Scholz, R.-D., McCaughrean, M. J., Lodieu, N., \& Kuhlbrodt, B. 2003, A\&A, 398, L29

Scholz, R.-D., Lehmann, I., Matute, I., \& Zinnecker, H. 2004, A\&A, 425,519

Subasavage, J. P., Henry, T. J., Hambly, N. C., Brown, M. A., \& Jao, W.-Ch. 2004, AJ, accepted [arXiv: astro-ph/0409505]

Teegarden, B. J., Pravdo, S. H., Hicks, M., et al. 2003, ApJ, 589, L51

Tinney, C. G. 1998, MNRAS, 296, L42

Tinney, C. G., \& Reid, I. N. 1998, MNRAS, 301, 1031 